\title{
Un nouveau procédé d'injection de vapeur : application à la mise au point d'un nouveau traitement thermique du lait
}

\author{
C Esnouf, E Abou Mansour \\ avec la collaboration technique de G Delance \\ Centre national du machinisme agricole du génie rural, des eaux et des forêts, \\ Division transformation thermique des produits agricoles, Parc de Tourvoie, \\ BP 121, 92164 Antony Cedex, France
}

(Reçu le 28 novembre 1989; accepté le 12 mars 1990)

\begin{abstract}
Résumé - Un nouveau dispositif d'injection de vapeur dans un liquide est présenté. L'injecteur exploite les instabilités d'interface liquide-vapeur créées par la force centrifuge lorsque la trajectoire du liquide est courbe. Une condensation rapide de la vapeur est obtenue, ce qui permet d'élever la température du liquide de $80^{\circ} \mathrm{C}$ à $160^{\circ} \mathrm{C}$ en $0,15 \mathrm{~s}$. L'application de ce dispositif au traitement thermique du lait est présentée. L'objectif est la mise au point d'un nouveau procédé de stérilisation préservant mieux les qualités organoleptiques que le traitement UHT, grâce à un traitement de $0,2 \mathrm{~s}$ ou 0,4 $s$ à $160^{\circ} \mathrm{C}$. Le lait traité est stérile, la qualité organoleptique est proche d'un lait pasteurisé, mais le taux de dénaturation des protéines solubles est plus élevé que prévu. Une conservation de longue durée à $30^{\circ} \mathrm{C}$ n'est fas possible en raison d'une protéolyse très importante, qui est attribuée à la fois à la plasmine et aux protéases des bactéries psychrotrophes, qui ne sont pas inactivées par le traitement. Des solutions sont proposées pour améliorer les performances obtenues ou mettre au point un nouveau produit, à conservation réfrigérée.
\end{abstract}

lait / stérilisation UHT / nouveau procédé / qualité / injection directe de vapeur / protéolyse / dénaturation des protéines solubles

Summary - A new process of direct steam injection: application towards a new thermal treatment of milk. A new direct steam injection process is presented; it uses the liquid-steam interface instabilities created by the centrifugal force in a curve of the liquid flow. A rapid steam condensation is obtained, so that the liquid temperature is raised from $80^{\circ} \mathrm{C}$ to $160^{\circ} \mathrm{C}$ in $0.15 \mathrm{~s}$. The application of this process to the thermal treatment of milk is also presented. The aim is to develop a new process of sterilization giving better organoleptic properties than UHT milk, thanks to treatment at $160^{\circ} \mathrm{C}$ during $0.2 \mathrm{~s}-0.4 \mathrm{~s}$. The treated milk is sterile, its organoleptic quality is close to that of pasteurized milk, but the whey protein denaturation is higher than expected. A long conservation period at $30{ }^{\circ} \mathrm{C}$ is not possible because of extended proteolysis, attributed to both plasmin and psychrotrophic bacteria proteases, which are not inactivated by the treatment. Solutions to improve the results obtained or to develop a new product, with a refrigerated conservation, are suggested.

milk / UHT sterilization / new process / quality / direct steam injection / proteolysis / whey protein denaturation 


\section{INTRODUCTION}

Le traitement à ultra-haute température (UHT) est un procédé de plus en plus répandu dans l'industrie agro-alimentaire, spécialement pour la stérilisation du lait.

On distingue en général 2 modes de chauffage UHT : le mode direct et le mode indirect. Dans le premier cas, le lait est mélangé avec le fluide chauffant (la vapeur d'eau en général), alors que dans le deuxième cas, une paroi sépare le lait du fluide chauffant (vapeur d'eau ou eau chaude sous pression). Le premier procédé donne un lait de meilleure qualité étant donné que l'échange de chaleur est excellent et autorise par conséquent des temps de chauffage très courts, mais il est plus coûteux en énergie (Burton, 1977; Perkin, 1985). En effet la vapeur récupérée à la détente, après stérilisation, nécessite des dispositifs coûteux pour être revalorisée, alors que l'échangeur à contre-courant permet à lui seul une récupération de chaleur de 80 à $90 \%$.

Pour améliorer la qualité du lait stérilisé, il faut préserver les protéines solubles du lait et éviter les modifications physicochimiques, tout en détruisant les bactéries et enzymes (protéases, lipases) qu'il contient. L'élévation de la température de traitement répond à cet objectif, car l'énergie d'activation des réactions indésirables est faible par rapport à celle des destructions microbiennes. C'est déjà cet effet qui est utilisé dans le traitement UHT, par rapport à la stérilisation classique.

$\mathrm{Si}$ on augmente encore la température, jusqu'à $160^{\circ} \mathrm{C}$ par exemple, l'écart est encore plus grand entre le temps nécessaire pour détruire les bactéries et les spores, et le temps maximum au bout duquel les modifications physico-chimiques se produisent. Pour les spores de Clostridium botulinum, par exemple, le temps de réduction décimale à $120^{\circ} \mathrm{C}, D 120$, est égal à $12 \mathrm{~s}$ et $z=10^{\circ} \mathrm{C}$ (Trossat, 1980) ( $z=$ augmentation de température qui divise par 10 le facteur $D$ ); on a donc, à $160^{\circ} \mathrm{C}$, un temps de réduction décimale de $1,2 \cdot 10^{-3} \mathrm{~s}$. On a donc 10 réductions décimales en $12 \mathrm{~ms}$, alors que le brunissement apparaît autour de $20 \mathrm{~s}$, que la destruction de $3 \%$ de thiamine, et un taux de $30 \%$ de dénaturation des protéines solubles ont lieu respectivement au bout de $4 \mathrm{~s}$ et $1 \mathrm{~s}$ (Lyster et Hillier, 1979; Cerf, 1982; Dannenberg, 1986).

Un traitement à $160^{\circ} \mathrm{C}$ de quelques dixièmes de seconde apparaît par conséquent bénéfique. $\mathrm{Ce}$ traitement, imaginé par le laboratoire de génie des procédés alimentaires de I'INRA, a été appelé stérilisation à hyper-haute température (HHT) (Trossat, 1980). Technologiquement, un tel traitement n'est possible que par un procédé d'injection directe de la vapeur, étant donné que l'échange thermique doit être très rapide.

Un injecteur devant répondre à ces objectifs a été mis au point à l'INRA (Trossat, 1980; Fabre, 1982). II s'agit d'un injecteur Chawla (Chawla, 1977) dans lequel la vapeur est introduite par 12 orifices percés de part et d'autre d'un cylindre à l'intérieur duquel s'écoule le liquide à réchauffer. La modification essentielle apportée est le remplacement du chambreur par un fort rétrécissement de la canalisation, à la sortie. L'originalité de cet injecteur réside en l'établissement d'un régime critique de vitesse dans l'ajutage, ce qui stabilise le débit de liquide, indépendamment des variations de pression à l'aval; cet ajutage remplace une vanne avant la détente sous vide. Cet injecteur permet une condensation totale de la vapeur avant l'ajutage et des débits de $200 \mathrm{l} / \mathrm{h}$ ont pu être ainsi chauffés à $150^{\circ} \mathrm{C}$. Cette température n'a cependant pas pu être dépassée dans l'état actuel du dispositif. 
L'objectif du présent article est :

- la présentation d'un injecteur permettant de traiter les liquides à $160^{\circ} \mathrm{C}$ et plus;

- l'étude de l'aptitude de ce traitement à la stérilisation du lait;

- la présentation des modifications physico-chimiques du lait traité selon le procédé HHT, par rapport à un lait ayant subi un traitement UHT;

- l'étude de l'aptitude à la conservation du lait obtenu selon le procédé HHT.

\section{MATÉRIEL ET MÉTHODES}

\section{L'injecteur}

L'injecteur a fait l'objet d'une prise de brevet $n^{\circ} 8117654$ en 1981, en France, ainsi qu'en Europe, aux États-Unis et au Japon. Le principe de l'injecteur est l'exploitation des instabilités d'interface entre 2 fluides en présence d'un champ d'accélération dirigé du fluide le plus pesant vers le fluide le moins pesant, dites instabilités de Rayleigh-Taylor et de Kelvin-Helmotz (Clift, 1978; Hetsroni, 1982).

Les bulles de vapeur sont obtenues dans le cas d'une perturbation importante par dislocation de l'interface liquide/vapeur; leur taille est d'autant plus petite que la longueur d'onde sera plus faible et donc que l'accélération sera plus importante. L'accélération est ici l'accélération centrifuge créée par le passage du liquide dans un coude et la vapeur est injectée à l'extérieur du coude (fig 1). L'accélération est perpendiculaire à l'interface et la trajectoire des bulles sous l'action de cette accélération est une traversée de la veine liquide jusqu'au rayon intérieur. Cette trajectoire permet au liquide d'être traité de façon homogène.

L'accélération ainsi créée étant importante (voir plus loin), les bulles sont de petite taille. La surface d'échange entre les 2 phases est ainsi très grande et la condensation de la vapeur; donc l'échange thermique, peut être très rapide.

D'un point de vue technologique, le coude est constitué par un ensemble bague-noyau démontable, la vapeur étant injectée à travers la bague par l'intermédiaire de fentes (fig 1). Par changement de la bague on peut disposer d'un nombre et d'une géométrie de fentes différentes.

Le coude a une section rectangulaire de $5 \mathrm{x}$ $12 \mathrm{~mm}$. Les tuyaux d'entrée et de sortie ont un diamètre intérieur de $8 \mathrm{~mm}$. Le débit de liquide variant de 250 à $500 \mathrm{l} / \mathrm{h}$, les caractéristiques de l'écoulement sont les suivantes :

- l'accélération centrifuge varie entre $185 \mathrm{~m} / \mathrm{s}^{2}$ et $740 \mathrm{~m} / \mathrm{s}^{2}$ soit des valeurs bien supérieures à l'accélération de la pesanteur;

- la turbulence de l'écoulement est assurée.

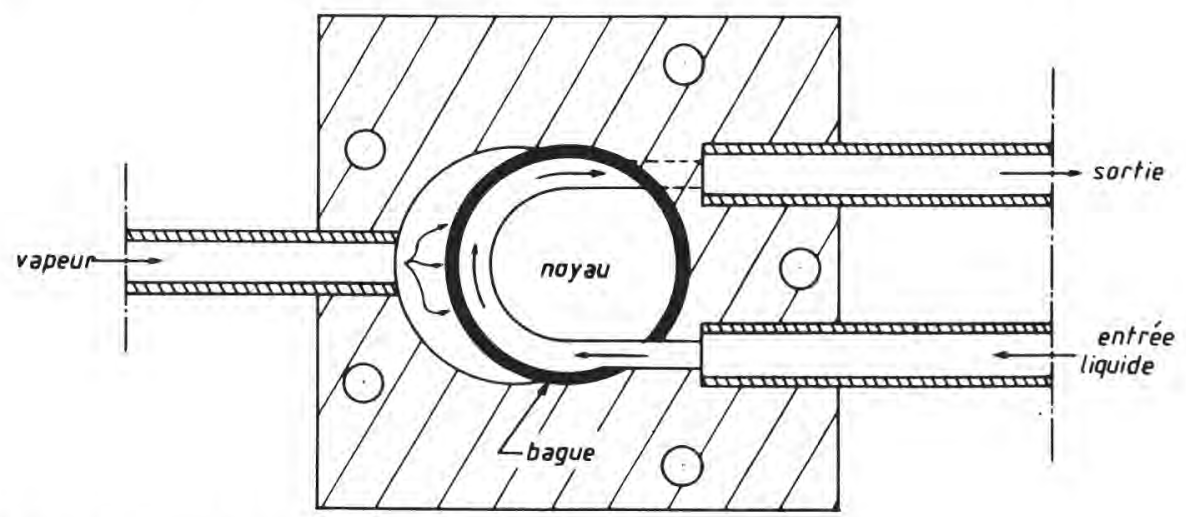

Fig 1. Vue en coupe de l'injecteur. Injector section view. 
Le nombre de Reynolds du liquide est de 30000 à 60000 pour l'eau à $80^{\circ} \mathrm{C}$.

\section{Dispositif expérimental}

La figure 2 représente le schéma général de l'installation sur laquelle se sont déroulés les essais. Le liquide est envoyé par une pompe centrifuge, du bac de lancement vers un échangeur à plaques qui joue le rôle de préchauffeur. Sortant de cet échangeur, le liquide est envoyé dans l'injecteur à l'aide d'une pompe volumétrique "Moineaun. Pour un débit de $250 \mathrm{l} / \mathrm{h}$, la vitesse du liquide est de $0,8 \mathrm{~m} / \mathrm{s}$ dans l'injecteur et de $1,4 \mathrm{~m} / \mathrm{s}$ dans les conduits d'entrée et de sortie. Le temps de séjour du liquide entre la fente d'injection de vapeur et la sortie de l'injecteur (considérée à la première mesure de température) est de $0,15 \mathrm{~s}$. Sous réserve que la condensation soit totale à la sortie, il s'agira du temps de montée à hyper-haute température.

L'injecteur est suivi d'une conduite de $8 \mathrm{~mm}$ de diamètre qui fait office de chambreur. Deux longueurs de chambreur $(25 \mathrm{~cm}$ et $50 \mathrm{~cm})$ ont été utilisées, qui correspondent à des temps de chambrage de 0,18 et de $0,35 \mathrm{~s}$ à $250 \mathrm{l} / \mathrm{h}$. Une vanne de contrepression (V1), qui garde le liquide sous pression, est disposée au bout de ce chambreur. Une autre vanne de contrepression (V2) est disposée au bout d'un deuxième chambreur en série dans lequel le liquide peut être gardé autour de $140^{\circ} \mathrm{C}$ (traitement UHT). Ceci peut permettre un traitement UHT du lait pendant une certaine phase de l'essai; pour le traitement HHT, la vanne V2 est complètement ouverte.

Les figures 3 et 4 montrent, sur un diagramme temps-température, l'évolution moyenne de la température d'une particule de lait traversant l'installation en fonction du temps. La première figure représente le traitement HHT, la deuxième le traitement UHT. Après une montée progressive jusqu'à $80^{\circ} \mathrm{C}$ dans le préchauffeur, le lait reste à cette température pendant $55 \mathrm{~s}$. II est ensuite porté à $160^{\circ} \mathrm{C}$ (HHT) ou à $140^{\circ} \mathrm{C}$ (UHT) en 0,15 s (temps de séjour dans l'injecteur) pour rester à cette température pendant en moyenne $0,4 \mathrm{~s}$ (HHT) ou pendant $28 \mathrm{~s}$ (UHT). Ce traitement UHT est excessif par rapport à un traitement UHT direct industriel $\left(140^{\circ} \mathrm{C}\right.$ pendant 5 à $\left.7 \mathrm{~s}\right)$, mais il était impossible de diminuer ce temps pour des raisons de disposition géométrique de l'expérience. Le lait UHT obtenu avec ce traitement est comparable à un lait UHT indirect $\left(15 \mathrm{~s}\right.$ à $140^{\circ} \mathrm{C}$ et une montée et descente en température lente). Le lait est refroidi très rapidement au niveau de la détente sous vide partiel jusqu'à la température de $70{ }^{\circ} \mathrm{C}$, et ensuite refroidi progressivement dans l'échangeur à eau glacée d'où il ressort à une température de l'ordre de $6^{\circ} \mathrm{C}$.

II faut noter que le palier de température à $110^{\circ} \mathrm{C}$ du diagramme HHT (fig 3 , en pointillé) est dû à la section de chambrage UHT qui ne s'est pas trouvée mise sous vide malgré l'ouverture complète de V2, cette vanne présentant une section de passage assez faible. Ce palier a été éliminé à partir de l'essai $n^{\circ} 7$ en changeant la vanne $\mathrm{V} 2$.

Le lait stérilisé est recueilli sous atmosphère stérile (hotte à flux laminaire) pour être conditionné (en flacons plastiques ou bouteilles de verre capsulées). Si le lait est gras, il doit passer par une phase d'homogénéisation (l'homogénéisateur est alors sous barrière stérile).

La vapeur est fournie par une chaudière à vaporisation instantanée "vapor" munie d'un brûleur à fuel, de $100 \mathrm{~kg} / \mathrm{h}$ de débit vapeur maximum. La vapeur est de qualité alimentaire après passage dans un filtre.

Pour ce qui concerne les mesures, les implantations des capteurs sont indiquées sur la figure 2. Pour pouvoir suivre les variations rapides de la température (T), surtout à la sortie de linjecteur, on a utilisé des sondes à faible temps de réponse, thermocouples K (Chromel, Alumel) de $0,25 \mathrm{~mm}$ de diamètre (Thermocoax). Tous les thermocouples ont été étalonnés de 0 à $200{ }^{\circ} \mathrm{C}$ sur un calibrateur Jofra 6005 . L'ensemble thermocouple + chaîne d'acquisition donne une incertitude absolue de $\pm 1^{\circ} \mathrm{C}$. Une mesure de débit liquide est réalisée à l'aide d'un débitmètre électromagnétique «Krohne" (mesure basée sur la loi de Faraday). Les capteurs de pression $(P)$ sont des capteurs à jauges résistives «JPB» et «SEDEME» de 0-25 bars et 0 50 bars.

Une saisie informatisée des données a permis de suivre les mesures de température et de pression (une saisie de toutes les voies toutes les 2, 3 s), à l'aide d'un micro-ordinateur Apple 


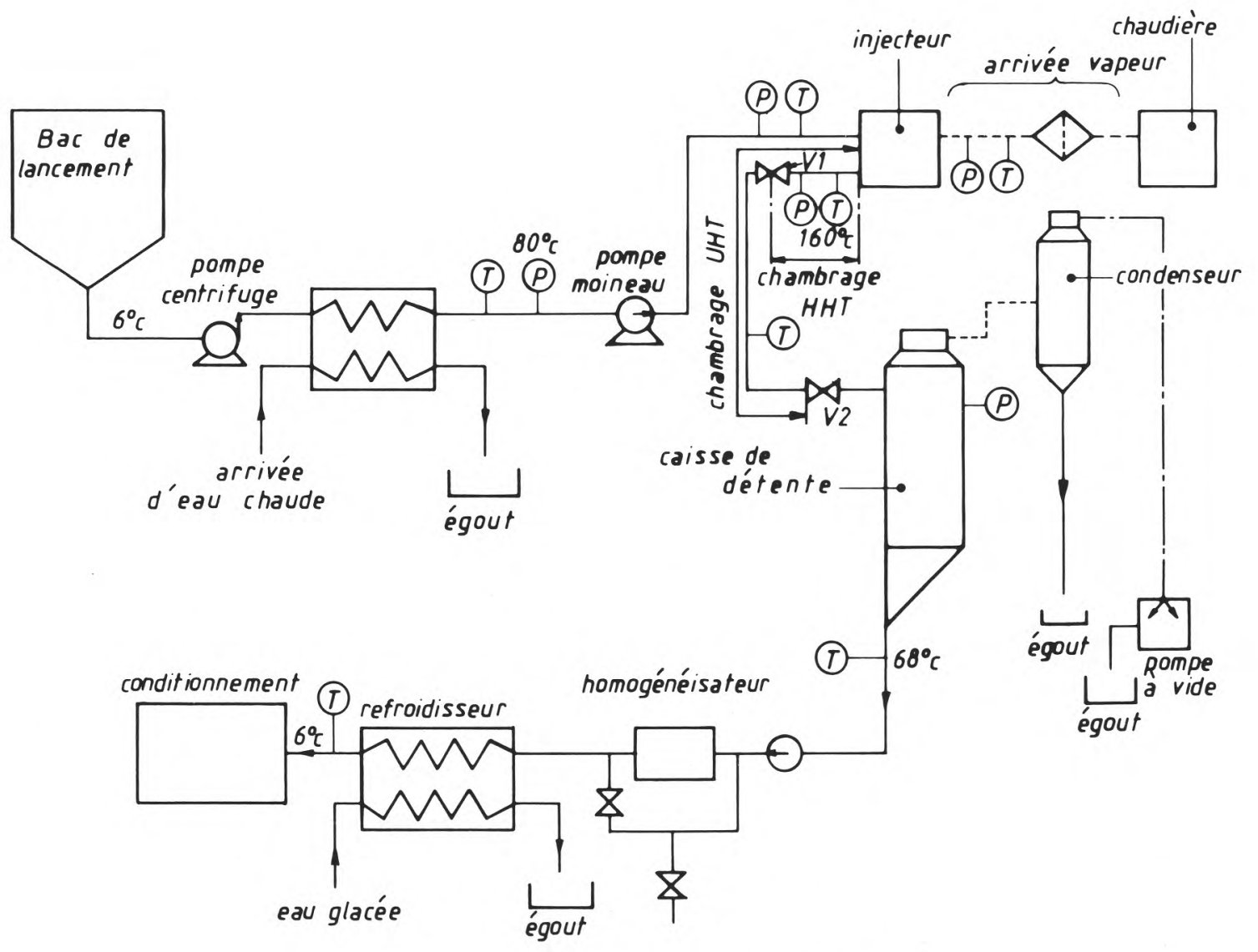

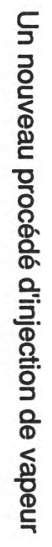

Fig 2. Schéma du dispositif expérimental. Experimental set-up. 


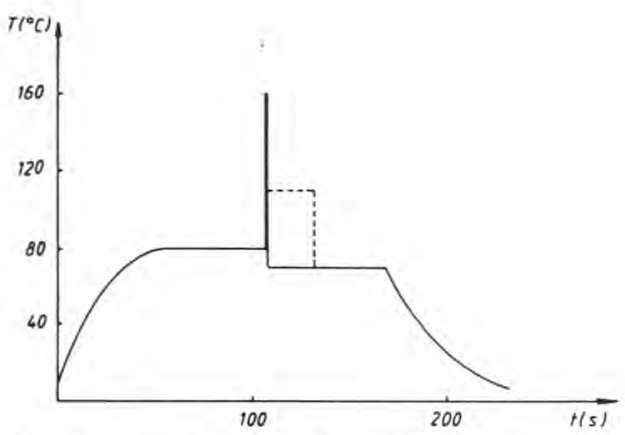

Fig 3. Diagramme temps-température du traitement HHT.

HHT treatment time-temperature diagram.

Ile muni d'un carte de conversion analogique numérique.

\section{Protocole expérimental des essais sur le lait}

Une première phase consiste en la stérilisation de l'installation par de l'eau à $160{ }^{\circ} \mathrm{C}$ pendant une heure. Après cette phase, le refroidisseur et la pompe à vide sont mis en fonctionnement et on produit du lait HHT (la température du lait à la sortie de l'injecteur est maintenue à $160^{\circ} \mathrm{C}$ ). Le réglage de la température de détente s'opère par une vérification rapide de l'extrait sec : on mesure le débit de sortie à l'aide d'un réservoir et d'un chronomètre et cette mesure est comparée à l'indication du débitmètre.

Durant la troisième phase, on produit du lait UHT : la température à la sortie de l'injecteur est diminuée jusqu'à $140^{\circ} \mathrm{C}$, ce en réduisant le débit de vapeur injecté, el, parallèlement, le temps de séjour moyen dans le chambreur est augmenté en fermant partiellement la vanne V2 et en ouvrant la vanne V1. On prépare, selon les essais, de 200 à 500 litres d'échantillon.

Les essais 1.1 à 5.1 (voir plus loin) ont été conditionnés sous flux laminaire dans des emballages plastiques stériles à fermeture par bouchons filetés. A partir de l'essai 6.1, le lait a également été conditionné dans des bouteilles en verre à fermeture par capsule (bouteilles de

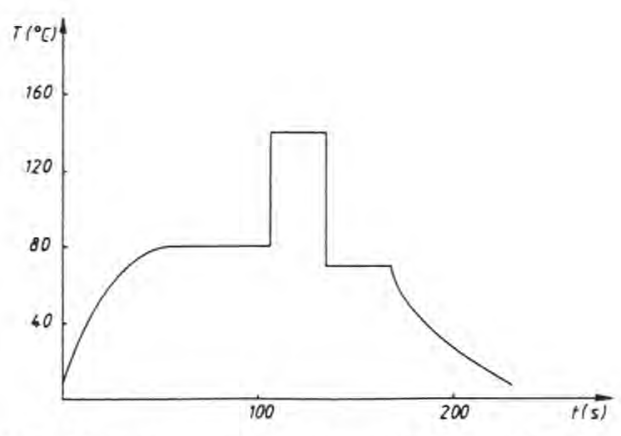

Fig 4. Diagramme temps-température du traitement UHT.

UHT treatment time-temperature diagram.

cidre), ce qui permettait de supprimer le volume d'air présent dans les emballages plastiques, soupçonné de causer des dégradations lors de la conservation à $30^{\circ} \mathrm{C}$.

Quatorze essais ont été réalisés. On détaille dans le tableau I les conditions exactes de ces essais. On a en général utilisé, par commodité, du lait thermisé en usine par chauffage à 63/ $65^{\circ} \mathrm{C}$ pendant 15 à $20 \mathrm{~s}$ (à $75^{\circ} \mathrm{C}$ pendant $30 \mathrm{~s}$ pour l'essai 14).

\section{Analyses}

Des analyses sont effectuées sur le lait de départ, le lait traité HHT, le lait traité UHT et éventuellement sur un lait UHT indirect du commerce. L'ensemble des analyses a été réalisé par la SODIMA (Société de développement et d'innovation des marchés agro-alimentaires) et I'ULN (Union laitière normande).

La stérilité a été contrôlée sur tous les essais. Pour les essais 1 à 4 , seuls l'extrait sec et la dégradation des protéines solubles ont été déterminés. Pour les essais suivants, un suivi de la conservation sur 10 à 12 semaines a été assuré, en particulier les essais $7,8,13,14$ ont donné lieu à des analyses détaillées, dont les procédures sont les suivantes :

- le contrôle de la stérilité est réalisé par une analyse bactériologique en germes totaux et germes thermorésistants réalisée selon la 
Tableau I. Conditions de traitement des essais sur le lait.

Experimental conditions of milk experiments.

\begin{tabular}{|c|c|c|c|c|c|c|}
\hline \multirow{2}{*}{$\begin{array}{l}\mathrm{N}^{\circ} \\
\text { essai }\end{array}$} & \multirow[t]{2}{*}{ type } & \multirow{2}{*}{$\begin{array}{l}\text { débit } \\
\text { lait } \\
(1 / \mathrm{h})\end{array}$} & \multirow{2}{*}{$\begin{array}{l}\text { lait de } \\
\text { départ }\end{array}$} & \multicolumn{3}{|c|}{$\begin{array}{l}\text { Traitement thermique } \\
\text { température - temps }\end{array}$} \\
\hline & & & & $\begin{array}{l}\text { préchauf- } \\
\text { fage }\end{array}$ & stérilisation & détente \\
\hline $\begin{array}{l}1.1 \\
1.2\end{array}$ & $\begin{array}{l}\text { HHT } \\
\text { UHT }\end{array}$ & 160 & $\begin{array}{l}\text { écrémé } \\
\text { thermisé }\end{array}$ & $79^{\circ} \mathrm{C} / 78 \mathrm{~s}$ & $\begin{array}{l}160^{\circ} \mathrm{C} / 0,6 \mathrm{~s} \\
145^{\circ} \mathrm{C} / 42 \mathrm{~s}\end{array}$ & $\begin{array}{l}110^{\circ} \mathrm{C} / 11 \mathrm{~s} \\
+68^{\circ} \mathrm{C} / 30 \mathrm{~s}\end{array}$ \\
\hline 2.1 & HHT & 220 & $"$ & $79^{\circ} \mathrm{C} / 57 \mathrm{~s}$ & $160^{\circ} \mathrm{C} / 0,41 \mathrm{~s}$ & $"$ \\
\hline $\begin{array}{l}3.1 \\
3.2\end{array}$ & $\begin{array}{l}\text { HHT. } \\
\text { UHT }\end{array}$ & 220 & $"$ & " & $\begin{array}{l}160^{\circ} \mathrm{C} / 0,41 \mathrm{~s} \\
140^{\circ} \mathrm{C} / 28 \mathrm{~s}\end{array}$ & $69^{\circ} \mathrm{C} / 30 \mathrm{~s}$ \\
\hline 4.1 & HHT & 260 & $"$ & $75^{\circ} \mathrm{C} / 29 \mathrm{~s}$ & $160^{\circ} \mathrm{C} / 0,35 \mathrm{~s}$ & $\begin{array}{l}110^{\circ} \mathrm{C} / 10 \mathrm{~s} \\
+65^{\circ} \mathrm{C} / 30 \mathrm{~s}\end{array}$ \\
\hline 5.1 & HHT & 250 & $"$ & $75^{\circ} \mathrm{C} / 30 \mathrm{~s}$ & $160^{\circ} \mathrm{C} / 0,35 \mathrm{~s}$ & " \\
\hline 6.1 & HHT & 250 & $\begin{array}{l}\text { demi-écrémé } \\
\text { thermi sé }\end{array}$ & " & " & " \\
\hline 7.1 & HHT & 250 & $\begin{array}{l}\text { après trai- } \\
\text { tement }\end{array}$ & $"$ & $"$ & \\
\hline $\begin{array}{l}8.1 \\
8.2 \\
8.3\end{array}$ & $\begin{array}{l}\quad \text { HHT } \\
\text { UHT } \\
\text { pasteu- } \\
\text { risé }\end{array}$ & $\begin{array}{l}250 \\
250 \\
250\end{array}$ & $\begin{array}{l}\text { écrémé } \\
\text { thermisé }\end{array}$ & $\begin{array}{l}73^{\circ} \mathrm{C} / 30 \mathrm{~s} \\
73^{\circ} \mathrm{C} / 30 \mathrm{~s} \\
73^{\circ} \mathrm{C} / 30 \mathrm{~s}\end{array}$ & $\begin{array}{c}160^{\circ} \mathrm{C} / 0,35 \mathrm{~s} \\
148^{\circ} \mathrm{C} / 30 \mathrm{~s} \\
\text { sans }\end{array}$ & \\
\hline $\begin{array}{l}9.1 \\
9.2\end{array}$ & $\begin{array}{l}\text { HHT } \\
\text { UHT }\end{array}$ & $\begin{array}{l}250 \\
250\end{array}$ & $\begin{array}{l}\text { entier } \\
\text { thermisé et } \\
\text { homogénéisé } \\
\text { au préalable }\end{array}$ & $\begin{array}{c}69^{\circ} \mathrm{C} / 30 \mathrm{~s} \\
"\end{array}$ & $\begin{array}{l}159^{\circ} \mathrm{C} / 0,35 \mathrm{~s} \\
140^{\circ} \mathrm{C} / 30 \mathrm{~s}\end{array}$ & \\
\hline $\begin{array}{l}10.1 \\
10.2\end{array}$ & $\begin{array}{l}\text { HHT } \\
\text { UHT }\end{array}$ & $\begin{array}{l}250 \\
250\end{array}$ & $\begin{array}{l}\text { entier } \\
\text { thermisé }\end{array}$ & $75^{\circ} \mathrm{C} / 30 \mathrm{~s}$ & $\begin{array}{l}160^{\circ} \mathrm{C} / 0,2 \mathrm{~s} \\
140^{\circ} \mathrm{C} / 30 \mathrm{~s}\end{array}$ & $65^{\circ} \mathrm{C} / 30 \mathrm{~s}$ \\
\hline $\begin{array}{l}11.1 \\
11.2\end{array}$ & $\begin{array}{l}\text { HHT } \\
\text { UHT }\end{array}$ & $\begin{array}{l}250 \\
250\end{array}$ & $"$ & $"$ & $"$ & \\
\hline $\begin{array}{l}12.1 \\
12.2\end{array}$ & $\begin{array}{l}\text { HHT } \\
\text { UHT }\end{array}$ & $\begin{array}{l}250 \\
250\end{array}$ & $"$ & $"$ & $"$ & \\
\hline $\begin{array}{l}13.1 \\
13.2\end{array}$ & $\begin{array}{l}\text { HHT } \\
\text { UHT }\end{array}$ & $\begin{array}{l}200 \\
200\end{array}$ & $\begin{array}{l}\text { entier } \\
\text { cru }\end{array}$ & $73^{\circ} \mathrm{C} / 30 \mathrm{~s}$ & $\begin{array}{l}157 \text { à } 161^{\circ} \mathrm{C} / 0,45 \mathrm{~s} \\
138 \text { à } 140^{\circ} \mathrm{C} / 30 \mathrm{~s}\end{array}$ & \\
\hline $\begin{array}{l}14.1 \\
14.2\end{array}$ & $\begin{array}{l}\text { HHT } \\
\text { UHT }\end{array}$ & $\begin{array}{l}200 \\
200\end{array}$ & $\begin{array}{l}\text { écrémé } \\
\text { thermisé }\end{array}$ & $75^{\circ} \mathrm{C} / 30 \mathrm{~s}$ & $\begin{array}{l}157 \text { à } 160^{\circ} \mathrm{C} / 0,45 \mathrm{~s} \\
140^{\circ} \mathrm{C} / 30 \mathrm{~s}\end{array}$ & \\
\hline
\end{tabular}


norme FIL 49 et en organismes psychrotrophes selon la norme FIL 132. Ce contrôle peut être répété en cours de conservation si des dégradations sont observées;

- pour la teneur en protéines solubles et protéines totales, le fractionnement est réalisé selon la méthode de Rowland, le dosage de l'azote étant réalisé selon la méthode de Kjeldahl (FIL, 1962) pour les analyses de la SODIMA, et selon la méthode de Berthelot sur un Technicon Auto-analyseur II pour les analyses de I'ULN;

- la matière grasse est déterminée par la méthode de Gerber;

- l'extrait sec est déterminé selon l'arrêté du 24 883 du JO du 271083 ;

- le contenu en ammoniaque est déterminé par une électrode spécifique ORION, étalonnée avec des concentrations connues de $\mathrm{NH} 4 \mathrm{Cl}$;

- le test de Ramsdell (ou test du phosphate), indicateur de la stabilité du lait, est réalisé selon Ray (1951);

- la gélification est suivie par une mesure de viscosité sur un rhéomètre Carrimed (cône plan), cône de $6 \mathrm{~cm}$ et $2^{\circ}$ d'angle. La viscosité est déduite de l'analyse de la courbe de montée et descente à un couple de $10 \mu \mathrm{N}$.m selon un modèle newtonien;

- une analyse de la composition du lait a été réalisée sur les derniers essais par une électrophorèse sur gel de polyacrylamide en présence de SDS, selon la méthode de Laemmli (1970), et en présence d'urée selon la méthode de $\mathrm{Ng}$ Kwai-Hang et Kroeker (1984).

\section{Dégustations}

L'objectif est de mettre en évidence des différences entre 2 ou 3 traitements thermiques sur la perception sensorielle du lait au niveau des consommateurs. On effectue un test de type duo-trio pour comparer 2 laits entre eux. On dispose à chaque fois d'un lait HHT et d'un lait UHT et dans un cas d'un lait frais pasteurisé.

Les échantillons sont dégustés en boxes à $20^{\circ} \mathrm{C}$, dans des gobelets en plastique blanc. On demande au dégustateur de retrouver l'échantillon identique au témoin. Pour un niveau de confiance de $90 \%$, il faut obtenir 12 bonnes réponses sur 14 afin de conclure à une différence significative entre 2 laits. On n'identifie pas les critères qui ont été retenus par le jury pour porter son jugement.

\section{RÉSULTATS ET DISCUSSION}

\section{Mise au point du dispositif sur l'eau}

On a observé une instabilité de fonctionnement : la pression (réponse la plus rapide) à la sortie de l'injecteur présentait un écart type de 0,5 bar, qui correspondait à un écart de $3,3^{\circ} \mathrm{C}$ sur la température (fig 5). Ceci résultait de la combinaison d'une instabilité à haute fréquence (période supérieure à la fréquence de saisie) et à basse fréquence (période $20 \mathrm{~s}$ ).

Pour la basse fréquence, le fonctionnement alternatif du brûleur de la chaudière était responsable de l'instabilité (allumage sur une consigne de pression basse et extinction à la consigne haute); on a donc envoyé une partie de la vapeur à l'air libre afin de faire fonctionner la chaudière à pleine puissance quelle que soit la quantité

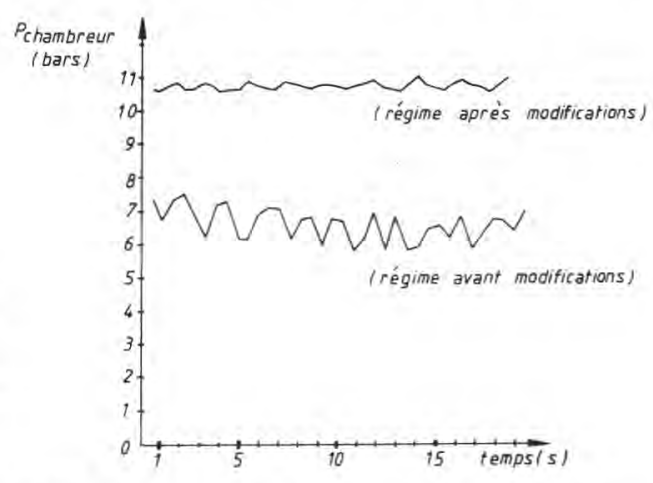

Fig 5. Variations de la pression du liquide dans le chambreur avant et après mise au point du dispositif.

Liquid pressure variations in the holding section before and after modifications. 
de vapeur utilisée par l'injecteur. Le brûleur fonctionnant alors en permanence assurait une pression constante de vapeur.

Les variations à haute fréquence sont dues, à l'origine, à la pompe d'injection et sont amplifiées par la condensation de la vapeur : en effet, la condensation de la vapeur entraîne une dépression locale, ce qui implique des oscillations de pression du liquide si celle-ci n'est pas constante; ces oscillations sont elles-mêmes répercutées sur la pression du circuit vapeur, ce qui amplifie le phénomène.

Nous avons donc augmenté les pertes de charge de la vapeur à l'injection, afin de rendre la pression de vapeur moins sensible aux variations de pression dans l'injecteur. Pour ce faire, on a réduit la zone d'injection à un seule fente. On a obtenu ainsi un régime très régulier (fig 5), l'écart type de la pression étant inférieur à 0,15 bar, ce qui représente $1^{\circ} \mathrm{C}$. Ce régime a été considéré comme satisfaisant pour les essais sur le lait.

\section{Stérilité}

La charge bactériologique des laits de départ est reportée dans le tableau II. II s'agit de laits de qualité et d'origine très variables, sauf pour les essais 13 et 14 où ils ont été sélectionnés pour leur bonne qualité bactériologique.

L'efficacité stérilisatrice théorique calculée sur la destruction de Clostridium botulinum selon les valeurs citées plus haut (Trossat, 1980) est de 330 réductions décimales pour un temps de séjour de $0,4 \mathrm{~s}$ à $160^{\circ} \mathrm{C}$ et 165 pour $0,2 \mathrm{~s}$ à $160^{\circ} \mathrm{C}$. On est donc, dans les 2 cas, largement au-delà de la sévérité requise (en général 12 réductions décimales sont suffisantes).

Sur 14 essais réalisés, 10 ont permis d'obtenir la stérilité totale ou partielle des échantillons. Les 4 essais non stériles ont pour origine des incidents expérimentaux (hotte à flux laminaire, arrêt de la chaudière...).

On peut donc considérer que l'injecteur de vapeur permet d'obtenir la stérilité du lait avec une montée de $80^{\circ} \mathrm{C}$ à $160^{\circ} \mathrm{C}$ en $0,15 \mathrm{~s}$ et un chambrage de $0,35 \mathrm{~s}$ ou $0,2 \mathrm{~s}$ à $160^{\circ} \mathrm{C}$. Ceci est logique car un traitement de $0,2 \mathrm{~s}$ à $115^{\circ} \mathrm{C}$ correspond à 50 réductions décimales des spores de Clostridium botulinum.

\section{Qualité du lait}

La qualité du lait a été appréciée par l'analyse de la teneur en protéines solubles et par des dégustations.

Tableau II. Analyse bactériologique des laits de départ.

Bacteriological analysis of milks before treatment.

\begin{tabular}{|c|c|c|c|c|c|c|c|c|}
\hline$n^{\circ}$ essai & \multicolumn{2}{|c|}{ germes } & $\begin{array}{l}\text { totaux } \\
55^{\circ} \mathrm{C}\end{array}$ & \multicolumn{3}{|c|}{$\begin{array}{c}\text { germes } \\
\text { thermorésistants } \\
30^{\circ} \mathrm{C} \quad 55^{\circ} \mathrm{C}\end{array}$} & \multicolumn{2}{|c|}{ psychrotrophes } \\
\hline 6 & 7 & 200 & 230 & & & 7 & & \\
\hline 8 & 15 & 000 & & 1 & 420 & 25 & & \\
\hline 9 & 211 & 000 & 230 & & 740 & & & \\
\hline 10 & 316 & 000 & 230 & 2 & 400 & 200 & & \\
\hline 13 & 67 & 000 & & 37 & 000 & 200 & 45 & 000 \\
\hline 14 & 47 & 000 & & & 200 & 0 & & 300 \\
\hline
\end{tabular}




\section{Teneur en protéines solubles}

Pour ce qui concerne la dénaturation des protéines solubles, on a comparé les résultats obtenus, avec un calcul effectué selon la méthode proposée par Cerf (1982). On suppose ainsi que les concentrations initiales des catégories de protéines solubles sont :

- $\alpha$-lactalbumine 1,2 g/l;

- $\beta$-lactoglobuline $2,7 \mathrm{~g} / \mathrm{l}$;

- sérum-albumine $0,25 \mathrm{~g} /$;

- immunoglobulines $0,65 \mathrm{~g} / \mathrm{l}$;

- protéoses-peptones 0,60 g/l;

soit au total $5,40 \mathrm{~g} / \mathrm{l}$. On fait les mêmes hypothèses que Cerf (1982) concernant l'utilisation des lois de dénaturation des différentes protéines établies expérimen- talement par Lyster et Hillier (1979) de $70^{\circ} \mathrm{C}$ à $150^{\circ} \mathrm{C}$. On suppose qu'il n'y a pas de dispersion des temps de séjour et on compte les temps de préchauffage et de détente supérieurs à $70^{\circ} \mathrm{C}$. Les résultats sont présentés dans le tableau III et la figure 6.

On constate qu'on a toujours une dénaturation inférieure dans le cas du traitement HHT par rapport au traitement de type UHT réalisé dans l'appareil.

Les pourcentages de dénaturation observés en UHT, de $49 \%$ à $68 \%$, correspondent aux valeurs obtenues par un traitement UHT indirect classique (un lait de départ a été traité en parallèle sur une ligne industrielle et a subi une dénaturation de $67,5 \%$ ). Ils présentent un écart de 2 à $20 \%$ en valeur absolue par rapport aux valeurs

\section{Pourcentage}

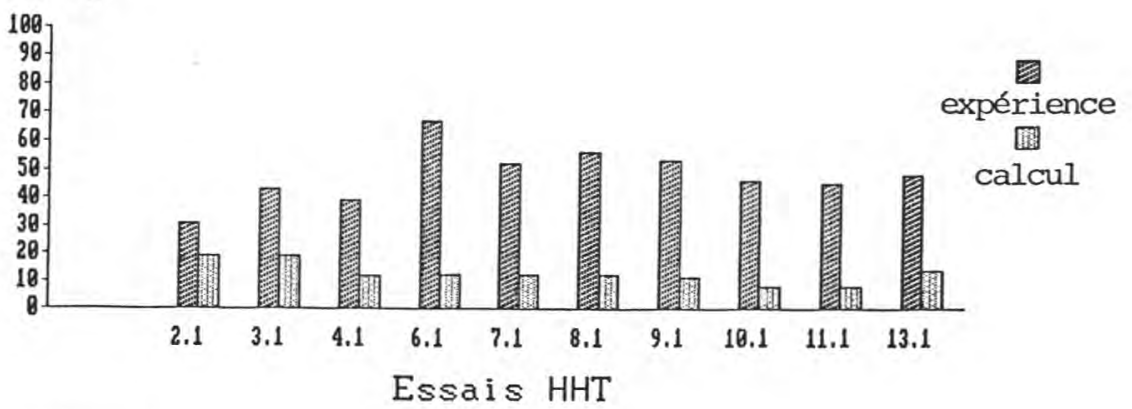

Pourcentage

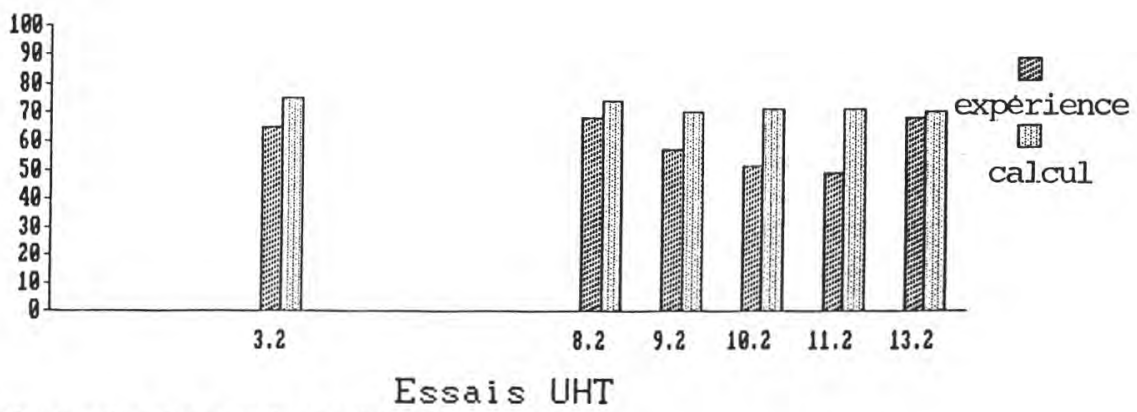

Fig 6. Dénaturation des protéines solubles.

Whey protein denaturation. 
calculées. Cet écart ne semble pas dû à I'hypothèse de concentrations fixes en différentes protéines retenues pour le calcul, car il n'est pas corrélé à la variation de ces concentrations telle qu'elle apparaît dans le tableau III.

Tableau III. Pourcentage de protéines solubles dénaturées. * Incertitude sur les caractéristiques $\mathrm{t}^{\circ} \mathrm{C} /$ temps de palier avant décompression complète.

Percentage of whey protein denaturation.

\begin{tabular}{|c|c|c|c|c|c|c|c|}
\hline \multirow{2}{*}{$\begin{array}{r}n^{\circ} \text { essai } \\
\text { type }\end{array}$} & \multicolumn{3}{|c|}{ Expérimentations } & \multicolumn{4}{|c|}{$\begin{array}{c}\text { Calcul } \\
\% \text { de dénaturation }\end{array}$} \\
\hline & $\begin{array}{c}\text { proteines } \\
\text { solubles } \\
\text { lait } \\
\text { départ } \\
(\mathrm{g} / 1)\end{array}$ & $\begin{array}{l}\text { proteines } \\
\text { solubles/ } \\
\text { protéines } \\
\text { totales } \\
\text { lait traité } \\
\%\end{array}$ & $\begin{array}{c}\text { dénaturation } \\
\text { par rapport } \\
\text { au lait de } \\
\text { départ } \\
\%\end{array}$ & $\begin{array}{l}\text { préchauf- } \\
\text { fage }\end{array}$ & $\begin{array}{l}\text { stérili- } \\
\text { sation }\end{array}$ & détente & Total \\
\hline $2.1 \mathrm{HHT}$ & 5,7 & 13 & 31 & 5,9 & 13 & $0-40 *$ & $18,9-58,9$ \\
\hline $3.1 \mathrm{HHT}$ & 6,9 & 13 & 43 & 5,9 & 13 & $0-40 *$ & $18,9-58,9$ \\
\hline $3.2 \mathrm{UHT}$ & 6,9 & 7,9 & 65 & 5,9 & 69 & 0,1 & 75 \\
\hline $4.1 \mathrm{HHT}$ & 6,9 & 14 & 39 & 0,7 & 11,5 & $0-40 *$ & $12,2-52,2$ \\
\hline $6.1 \mathrm{HHT}$ & 5,7 & 5,7 & 67 & 0,7 & 11,8 & - & 12,5 \\
\hline $7.1 \mathrm{HHT}$ & 6,5 & 9,7 & 52 & 0,7 & 11,8 & - & 12,5 \\
\hline $8.1 \mathrm{HHT}$ & 6 & 8,2 & 56 & 0,4 & 11,8 & - & 12,2 \\
\hline $8.2 \mathrm{UHT}$ & 6 & 6 & 68 & 0,4 & 73,8 & - & 74,2 \\
\hline 8.3 past & 6 & 14,3 & 24 & 0,4 & - & - & 0,4 \\
\hline $9.1 \mathrm{HHT}$ & 4,3 & 9 & 53 & 0,13 & 11,5 & - & 11,6 \\
\hline $9.2 \mathrm{UHT}$ & 4,3 & 8,7 & 57 & 0,13 & 70,2 & - & 70,4 \\
\hline $10.1 \mathrm{HHT}$ & 5,5 & 9,1 & 46 & 0,8 & 7,2 & - & 8 \\
\hline 10.2 UHT & 5,5 & 8,2 & 51 & 0,8 & 70,2 & - & 71 \\
\hline $11.1 \mathrm{HHT}$ & 5,4 & 9,2 & 45 & 0,8 & 7,2 & - & 8 \\
\hline $11.2 \mathrm{UHT}$ & 5,4 & 8,4 & 49 & 0,8 & 70,2 & - & 71 \\
\hline $12.1 \mathrm{HHT}$ & \multirow{2}{*}{\multicolumn{2}{|c|}{ non disponible }} & & 0,8 & 7,2 & - & 8 \\
\hline $12.2 \mathrm{UHT}$ & & & & 0,8 & 70,2 & - & 71 \\
\hline $13.1 \mathrm{HHT}$ & 8,6 & 14,4 & 48 & 0,4 & $13,3-14,3$ & - & 14 \\
\hline $13.2 \mathrm{UHT}$ & 8,6 & 8,9 & 68 & 0,4 & $69,2-70,2$ & - & 70 \\
\hline $14.1 \mathrm{HHT}$ & 5,6 & \multirow{2}{*}{\multicolumn{2}{|c|}{ non disponible }} & 1,5 & $13,3-14$ & - & 15 \\
\hline $14.2 \mathrm{UHT}$ & 5,6 & & & 1,5 & 70,2 & - & 70 \\
\hline
\end{tabular}


Dans le cas du lait HHT, les valeurs expérimentales de dénaturation, de $31 \%$ à $67 \%$, sont relativement dispersées et très éloignées des valeurs calculées (de 8 à $19 \%)$. On n'a pas d'amélioration significative pour un traitement de $0,2 \mathrm{~s}$ au lieu de 0,35 s (essais 10 et 11). On n'observe pas non plus d'influence de la teneur en matières grasses.

Plusieurs hypothèses peuvent être avancées concernant l'écart entre le calcul et les valeurs expérimentales, à savoir l'influence du préchauffage, l'influence du choc thermique, une dispersion des temps de séjour dans l'injecteur, et l'incertitude de l'extrapolation du modèle hors de son domaine de validité.

Pour ce qui concerne l'influence du préchauffage, l'échantillon 8.3 est significatif, puisqu'il n'a subi que l'étape de préchauffage $\left(30 \mathrm{~s}\right.$ à $\left.73^{\circ} \mathrm{C}\right)$. Cet échantillon présente un pourcentage de dénaturation de $24 \%$ par rapport au lait de départ, alors que le modèle donne $0,4 \%$. On a donc, à ce niveau, un écart très important, le modèle négligeant semble-t-il les cinétiques de dénaturation à basse température. Toutefois, même en tenant compte de cette différence, il reste un écart significatif à expliquer.

Pour ce qui concerne l'influence d'un choc thermique, le modèle a été utilisé par Daudin et Cerf (1977) entre $110^{\circ} \mathrm{C}$ et $125^{\circ} \mathrm{C}$ sur des injecteurs UHT directs à condensation de vapeur et il a été trouvé une corrélation à $\pm 5 \%$ des résultats expérimentaux sur le modèle. On ne peut donc pas retenir l'influence du choc thermique pour expliquer l'écart observé.

II est possible que l'écart soit dû à une dispersion des temps de séjour dans la section HHT, dispersion qui n'a pas été mesurée. Dans le cas où une partie du lait resterait en température pendant une période plus longue que le temps moyen, le tableau IV donne une indication des pourcentages de dénaturation correspondants : si la moitié du lait séjournait $2 \mathrm{~s}$ à $160^{\circ} \mathrm{C}$, on aurait en moyenne $18 \%$ de dénaturation supplémentaire sur l'ensemble du lait.

La dernière hypothèse pour expliquer cet écart est l'incertitude qui réside dans l'extrapolation à $160^{\circ} \mathrm{C}$ d'un modèle basé sur des expérimentations limitées à $150^{\circ} \mathrm{C}$ et corrélé jusqu'à $125^{\circ} \mathrm{C}$ seulement. Or selon Lyster cité par Walstra et Jenness (1984), la $\beta$-lactoglobuline, qui représente la moitié des protéines solubles, subit une dénaturation supplémentaire à partir de $140^{\circ} \mathrm{C}$ (ce qui est mis en évidence par un pic endothermique dans l'analyse calorimétrique) et cette dénaturation supplémentaire n'est pas prise en compte dans le modèle. Cependant, cette observation n'est pas mise en évidence dans les travaux de Dannenberg et Kessler (1988) effectués jusqu'à $150^{\circ} \mathrm{C}$. Rien ne nous permet donc de conclure quant à cet effet dans le cas du traitement HHT.

En conclusion, seules des expériences complémentaires permettraient une interprétation sûre du phénomène (étude de la dénaturation à basse température et mesure de la DTS en particulier).

\section{Dégustation}

Pour ce qui concerne les dégustations, 6 épreuves DUO/TRIO ont été effectuées :

- lors de 2 séries d'essais sur du lait écrémé (8.1 (HHT), 8.2 (UHT), 8.3 et 14.1

Tableau IV. Influence du temps de séjour à $160^{\circ} \mathrm{C}$ (obtenu par le calcul) sur le pourcentage de dénaturation des protéines solubles.

Time of treatment $\left(160^{\circ} \mathrm{C}\right.$ ) influence on whey protein denaturation.

\begin{tabular}{lrrrrr} 
temps (s) & 0,4 & 1 & 2 & 4 & 10 \\
dénaturation (\%) & 12 & 24 & 36 & 48 & 64 \\
\hline
\end{tabular}


(HHT), 14.2 (UHT)), les juges ont isolé de façon significative le lait HHT par rapport au lait UHT, ainsi que le lait UHT par rapport au lait pasteurisé; par contre, aucune différence significative n'était notée entre le lait HHT et le lait pasteurisé;

- lors d'une autre série d'essais portant sur du lait entier (13.1 (HHT) et 13.2 (UHT) après 20 jours de conservation à $7^{\circ} \mathrm{C}$ et $20^{\circ} \mathrm{C}$ ), ces résultats n'ont pas été retrouvés. II semble donc que l'écart ait été masqué par la matière grasse.

Aucune préférence marquée n'a été notée par les dégustateurs : le lait UHT présente un léger goût de cuit et le lait HHT un goût de lait plus fort.

On peut déduire de ces dégustations que le traitement HHT permet bien d'obtenir une amélioration de la qualité par rapport au traitement UHT, amélioration le rapprochant d'un lait pasteurisé. Cet effet semble cependant masqué par la matière grasse, ce qui limiterait l'application du procédé. Cet effet doit être confirmé par des tests plus nombreux.

\section{Conservation du lait}

On n'a observé aucune gélification sur l'ensemble des échantillons conservés au froid ou à température ambiante pendant 3 mois (la gélification est une évolution classique des laits UHT). L'extrait sec n'évolue pas. Par contre, le pourcentage de protéines solubles, l'indice de Ramsdell, et le taux d'ammoniaque évoluent.

Pour ce qui concerne le taux d'ammoniaque, on observe sur la figure 7 , une augmentation de ce taux de 6-8 ppm à 18-20 ppm, sans distinction toutefois entre laits HHT et UHT. Cette augmentation est classique dans le cas du lait UHT, mais elle est plus limitée en général (13 ppm au bout de 4 mois) selon Helaine (1981). Elle représente une décomposition chimique ou une protéolyse. Dans notre cas, elle n'est pas représentative de la protéolyse qui est très différente pour I'HHT et I'UHT, comme nous le verrons plus loin. La valeur du taux d'ammoniaque est peu élevée juste après traitement, ce qui est naturel puisque le lait est dégazé lors de la mise sous vide.

Pour ce qui concerne l'indice de Ramsdell, significatif de la stabilité du lait à la chaleur, on observe sur la figure 8 , une augmentation très nette de l'indice, due au traitement de stérilisation, c'est-à-dire une augmentation de la stabilité. Cette stabilité est plus importante pour les laits UHT que pour les laits HHT. En cours de conservation, l'écart se creuse puisque la stabilité des laits HHT diminue alors que la stabilité des UHT est constante (un lait commercial étudié en comparaison montre une évolution UHT classique). Cet indice de Ramsdell est considéré comme un indicateur de la protéolyse en cours de conservation du lait UHT (Helaine, 1981). En particulier, dans le cas oủ l'indice est, après 4 mois, inférieur à $50 \%$ de sa valeur après traitement, le lait est en voie de protéolyse et cette protéolyse est nette si cette diminution est atteinte au bout d'un mois ( $\mathrm{He}$ laine, 1981). Dans le cas de I'UHT, cet indice réagit plus vite que le taux de NPN.

Dans notre cas, on a diminution de l'indice à $50 \%$ de sa valeur au bout d'un mois et demi puis stabilisation. Or, comme nous allons le voir, les laits HHT sont soumis à une très forte protéolyse, qui se prolonge au-delà de cette date. L'indice de Ramsdell est donc un indicateur de protéolyse, mais il est peu approprié au traitement $\mathrm{HHT}$, car il ne descend pas à des valeurs très faibles qui indiqueraient une forte protéolyse. II est probable que la protéolyse observée est différente de la protéolyse classique des laits UHT. 


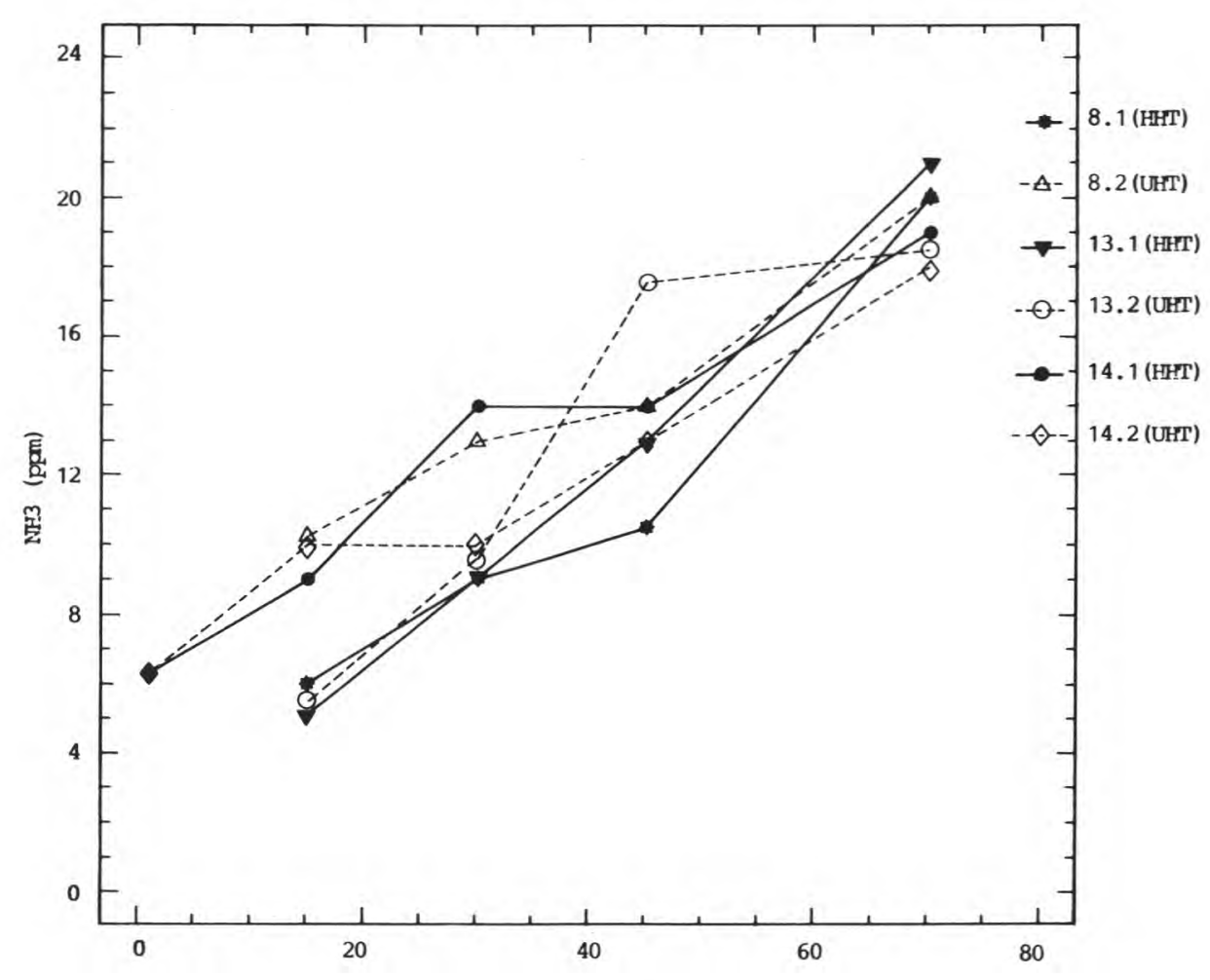

Jours de conservation

Fig 7. Conservation du lait : évolution de la teneur en ammoniaque. Essais HHT et UHT. Milk storage : $\mathrm{NH}_{3}$ evolution in HHT and UHT experiments.

L'évolution la plus significative concerne le taux de caséine du lait. Les premiers essais réalisés ont montré, après stockage du lait un mois à $30^{\circ} \mathrm{C}$, une modification de la couleur et une transparisation progressive, aboutissant à un liquide transparent et très amer (ressemblant à un lactosérum de fromagerie). Un suivi plus précis sur 6 à 12 semaines, en stockage réfrigéré ou à température ambiante, a alors été réalisé sur les essais $7,8,9,10,13,14$. L'essai 7 n'a, lui, été conservé qu'à $30^{\circ} \mathrm{C}$.

Pour la conservation à $30^{\circ} \mathrm{C}$, on observe sur la figure 9 que le taux de pro- téines solubles des laits HHT augmente au cours du temps, de façon très dispersée, puisque l'essai 7 n'est pas touché, les essais 9 et 10 le sont peu et les essais 13 et 14 vont jusqu'à 45 à $55 \%$ de protéines solubles. On a donc une protéolyse de la caséine.

Afin de connaître l'origine de cette protéolyse et en particulier savoir si elle est due à des enzymes ou à une contamination bactérienne, un ensemencement sur milieu PCA (Plate Count Analysis) a été réalisé à $12,20,33$ et 47 jours de conservation : il n'a montré aucune croissance bac- 


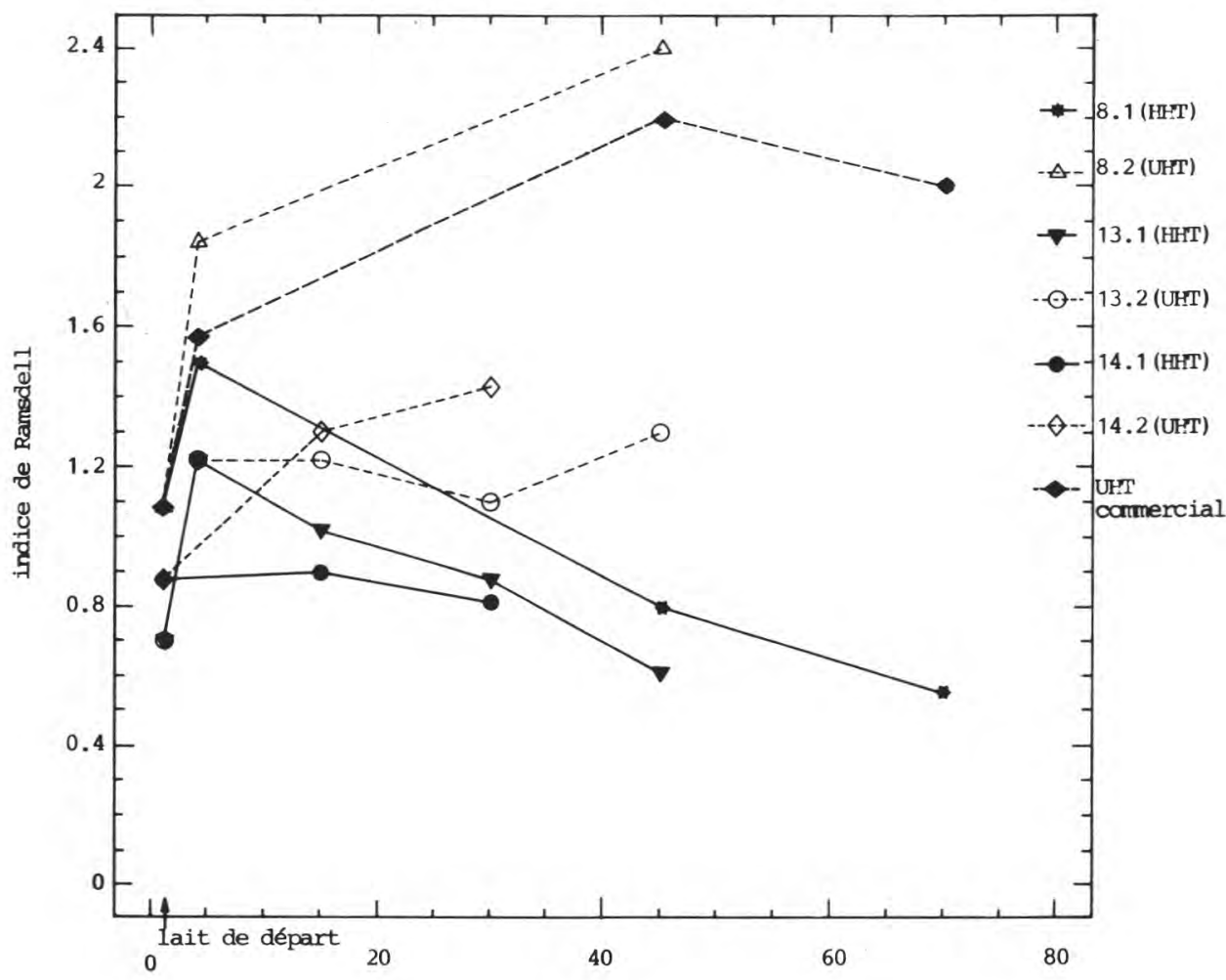

Jours de conservation

Fig 8. Conservation du lait : évolution de l'indice de Ramsdell. Essais HHT et UHT.

Milk storage : Ramsdell evolution in HHT and UHT experiments.

térienne. L'activité est donc imputée à des enzymes protéolytiques qui n'auraient pas été inactivées par le traitement.

Par comparaison, les figures 10 et 11 montrent que les laits UHT n'ont pas subi cette protéolyse et qu'ils se comportent de ce point de vue comme un lait UHT indirect classique (lait commercial témoin).

Les enzymes protéolytiques responsables de cette évolution peuvent être la plasmine naturelle du lait ou des enzymes d'origine microbienne, les protéases des bactéries psychrotrophes. En effet, il est connu que dans le cas des traitements UHT ces 2 types d'enzymes ne sont pas entièrement inactivées.

Pour ce qui concerne la plasmine, il est connu (Walstra et Jenness, 1984) que même un traitement de quelques secondes à $150^{\circ} \mathrm{C}$ n'en permet pas l'inactivation. D'après ces auteurs, on peut estimer un temps de réduction décimale de 1 $\min$ à $100^{\circ} \mathrm{C}$ et un facteur $z$ de 23 , ce qui donne un temps de réduction décimale de $1,1 \mathrm{~s}$ à $140^{\circ} \mathrm{C}$ et $0,15 \mathrm{~s}$ à $160^{\circ} \mathrm{C}$. Deux réductions décimales nécessitent donc $0,3 \mathrm{~s}$ 


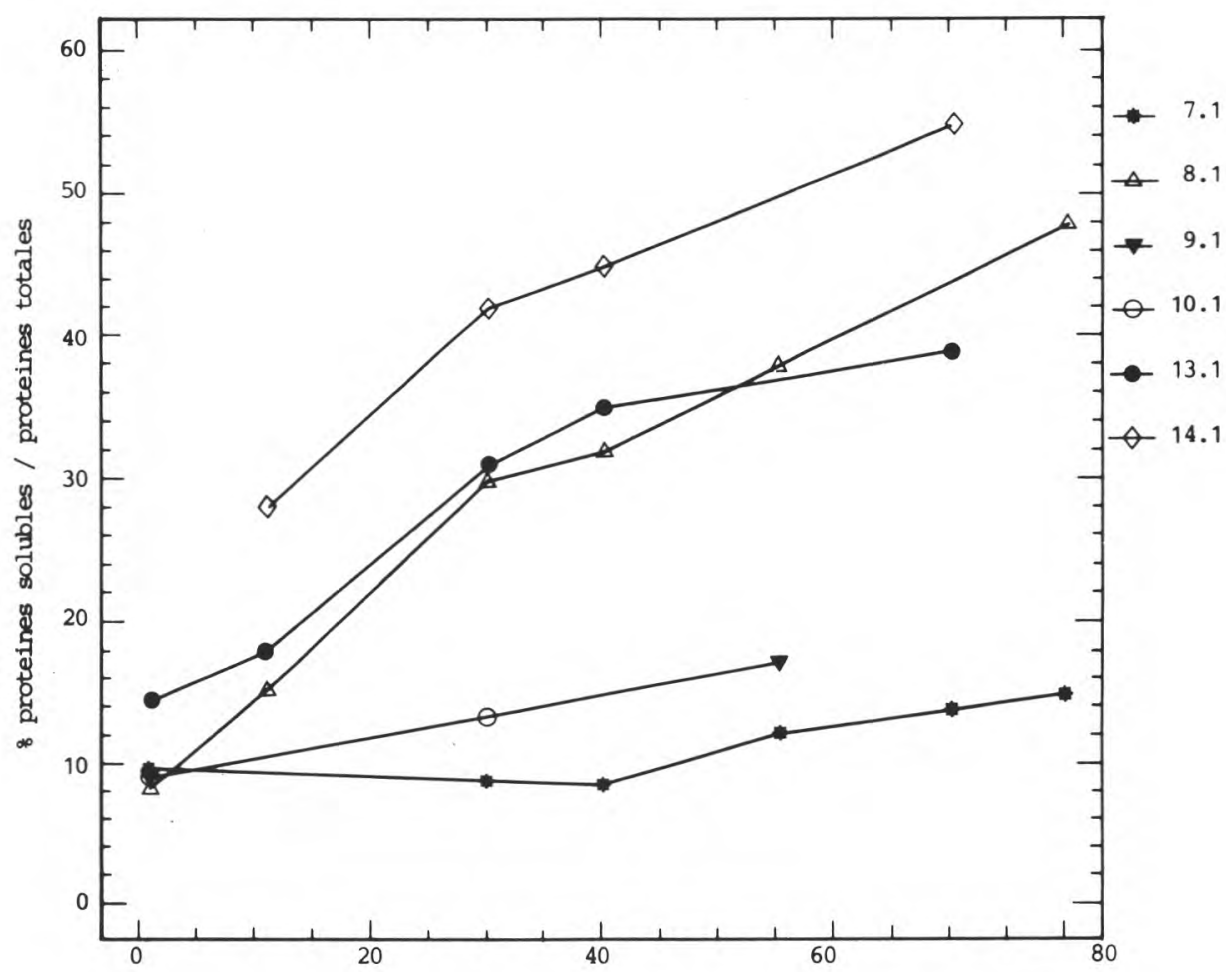

Jours de conservation

Fig 9. Conservation du lait HHT à 30 degrés : évolution des protéines solubles.

Milk storage at $30^{\circ} \mathrm{C}$ : whey protein evolution.

à $160{ }^{\circ} \mathrm{C}$, ce qui rend les conditions du traitement HHT tout juste satisfaisantes. II est à noter que la plasmine n'hydrolyse pas la caséine $k$ (Hill, 1988).
Pour ce qui concerne les protéases des bactéries psychrotrophes, le tableau V donne, selon diverses sources, la résistance à la température des protéases de

Tableau V. Résistance à la chaleur des protéases de bactéries psychrotrophes.

Heat resistance of psychrotrophic proteases.

\begin{tabular}{|l|c|c|c|c|}
\hline Microorganisme & $\mathrm{T}^{\circ} \mathrm{C}$ & $\begin{array}{c}\mathrm{D} \\
(\mathrm{min})\end{array}$ & $\mathrm{Z}$ & $\begin{array}{c}\text { temps pour 1 réduction } \\
\text { décimale à 160 }\end{array}$ \\
\hline P fluorescens & 120 & 4 & 20 & 2,4 \\
Pseudomonas & 149 & 1,5 & 32,5 & 41 \\
" & 150 & 1,7 & 32 & 50 \\
" & 150 & 0,5 & 32,5 & 15 \\
Pluorescens & 130 & 11 & 34,5 & 89 \\
" & 150 & 27 & 28 & 700 \\
\hline
\end{tabular}




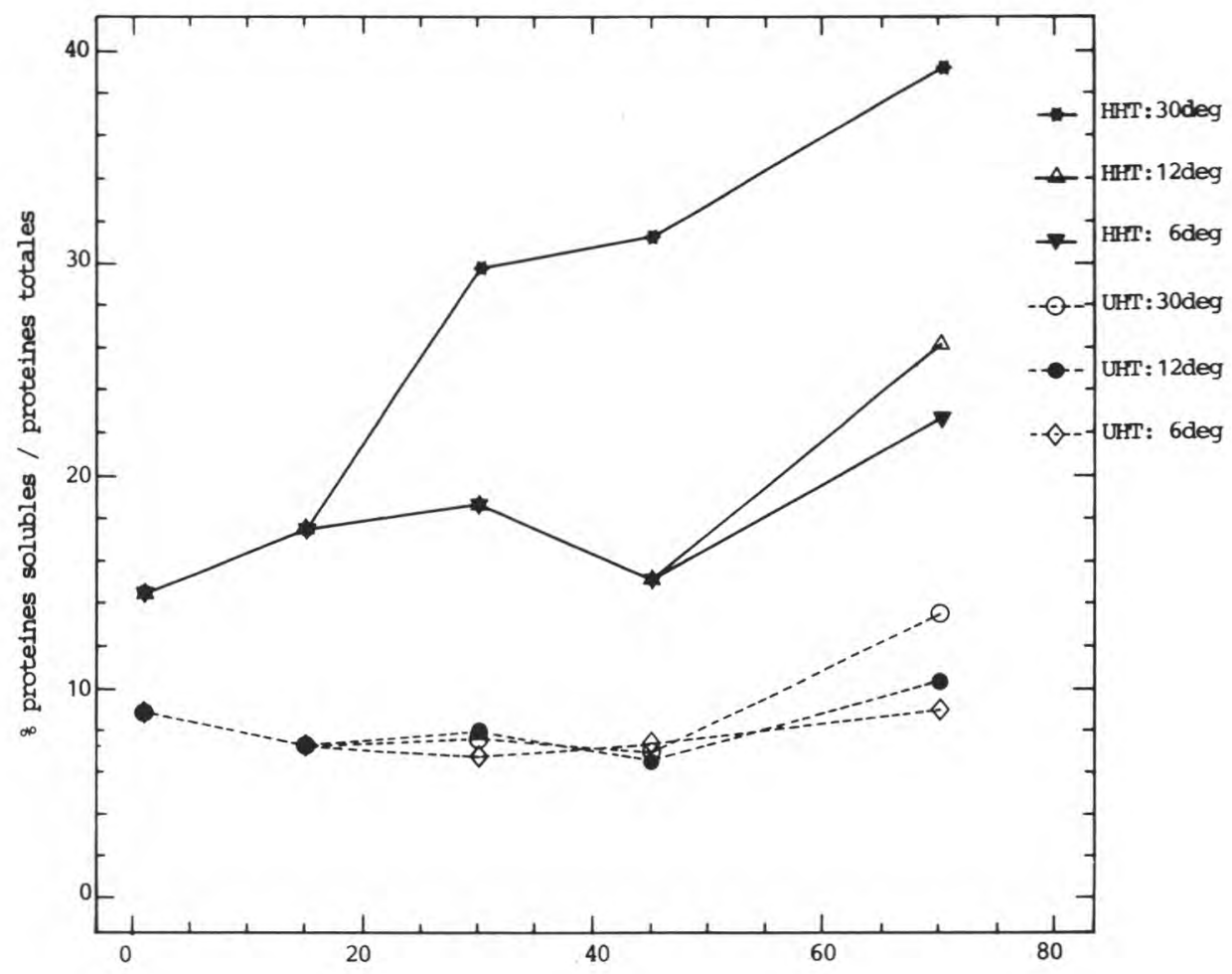

Jours de conservation

Fig 10. Influence du type de traitement et de la température de conservation. Essai 13. Influence of treatment characteristics and storage temperature. Experiment No 13.

différentes souches et le résultat de notre extrapolation, supposée linéaire, à $160^{\circ} \mathrm{C}$. II est alors clair que les $0,4 \mathrm{~s}$ du traitement HHT sont nettement insuffisantes pour assurer l'inactivation de ces protéases.

II existe 3 types de solution pour remédier à l'action de ces enzymes : une conservation au froid après traitement (Hill, 1988), un tri du lait afin de n'utiliser qu'un lait peu contaminé en bactéries psychrotrophes, soit moins de $10^{5}$ germes $/ \mathrm{ml}$ de psychrotrophes (Helaine, 1981) et un traitement d'inactivation à basse température
(Hill, 1988). Seules les 2 premières solutions ont pu être testées au cours du présent travail.

Concernant la première solution, un stockage réfrigéré du lait a été réalisé sur les essais 13 et 14 . Les figures 10 et 11 montrent effectivement une influence significative de la température de conservation sur la protéolyse. A $6{ }^{\circ} \mathrm{C}$ et, dans une moindre mesure, $12{ }^{\circ} \mathrm{C}$, le lait n'est pas déstabilisé avant. 45 jours. On peut donc considérer que le traitement HHT autorise la production d'un lait de bonne qualité se 


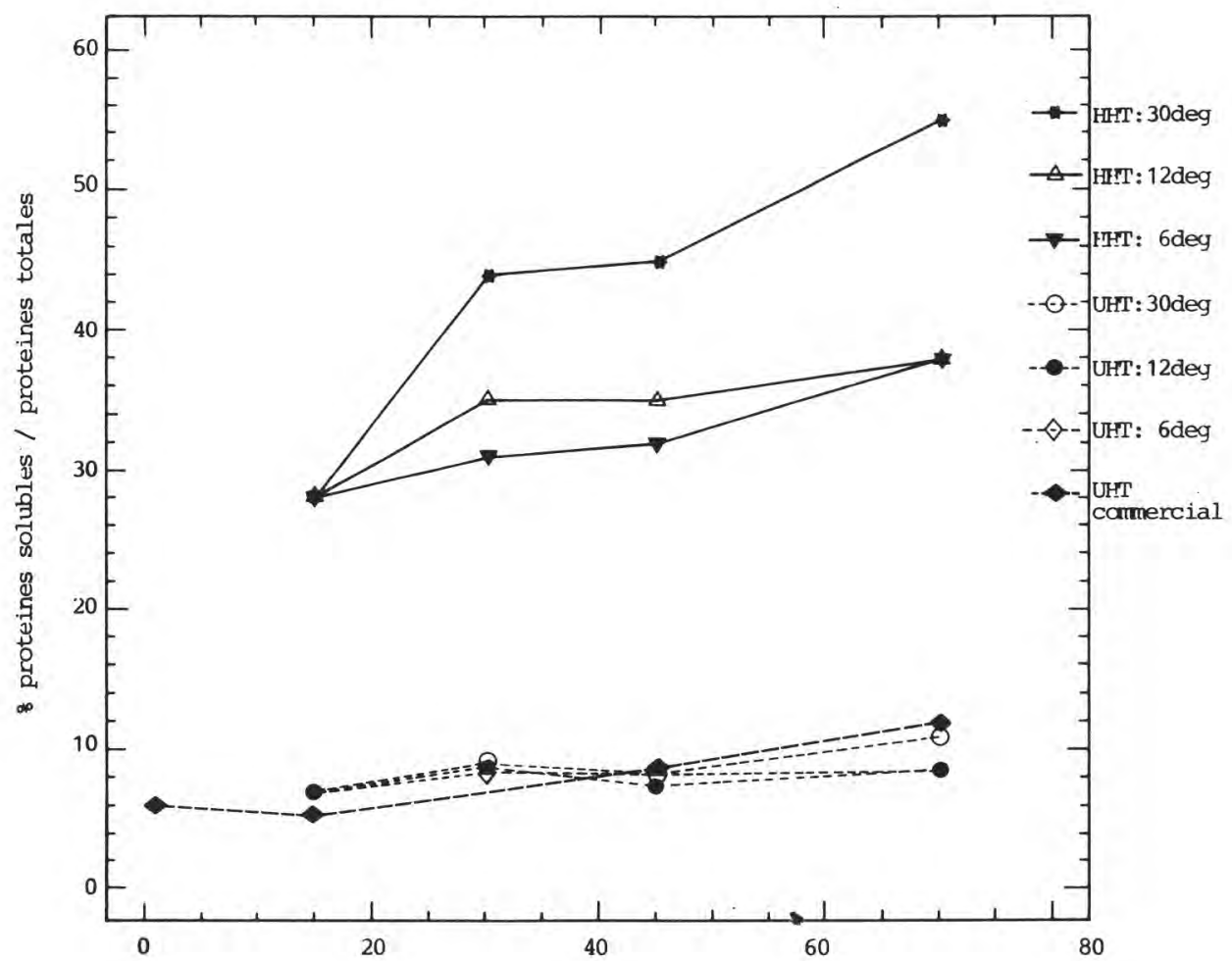

Jours de conservation

Fig 11. Influence du type de traitement et de la température de conservation. Essai 14. Influence of treatment characteristics and storage temperature. Experiment No 14.

conservant au froid pendant un mois à un mois et demi.

Concernant la seconde solution, un tri du lait a été effectué pour réaliser ces essais 13 et $14:$ le lait 13 contenait moins de 50000 germes $/ \mathrm{ml}$ totaux et le lait 14 moins de 40000 germes psychrotrophes/ ml.

On constate sur les figures 10 et 11 que la protéolyse à $30{ }^{\circ} \mathrm{C}$ est néanmoins très significative, puisqu'elle atteint $55 \%$ en 70 jours pour l'essai 14. Ceci conduirait à penser que l'action de la plasmine est prépondérante.
Afin d'éclairer ce point, un suivi de la composition du lait 13 en cours de dégradation a été réalisé par électrophorèse. La figure 12 montre que les caséines $\alpha_{\mathrm{s}}$ et $\beta$ sont dégradées au profit de la caséine $\gamma$ et de peptides et ce, avant la caséine $k$.

Puisque la plasmine ne dégrade pas la caséine $k$, ceci indique que la plasmine est très probablement le responsable essentiel de la protéolyse. La figure 13, qui présente l'évolution jusqu'à 45 jours montre cependant que la caséine $k$ finit par être atteinte, ce qui conduit à penser que les protéases bactériennes interviennent également, 

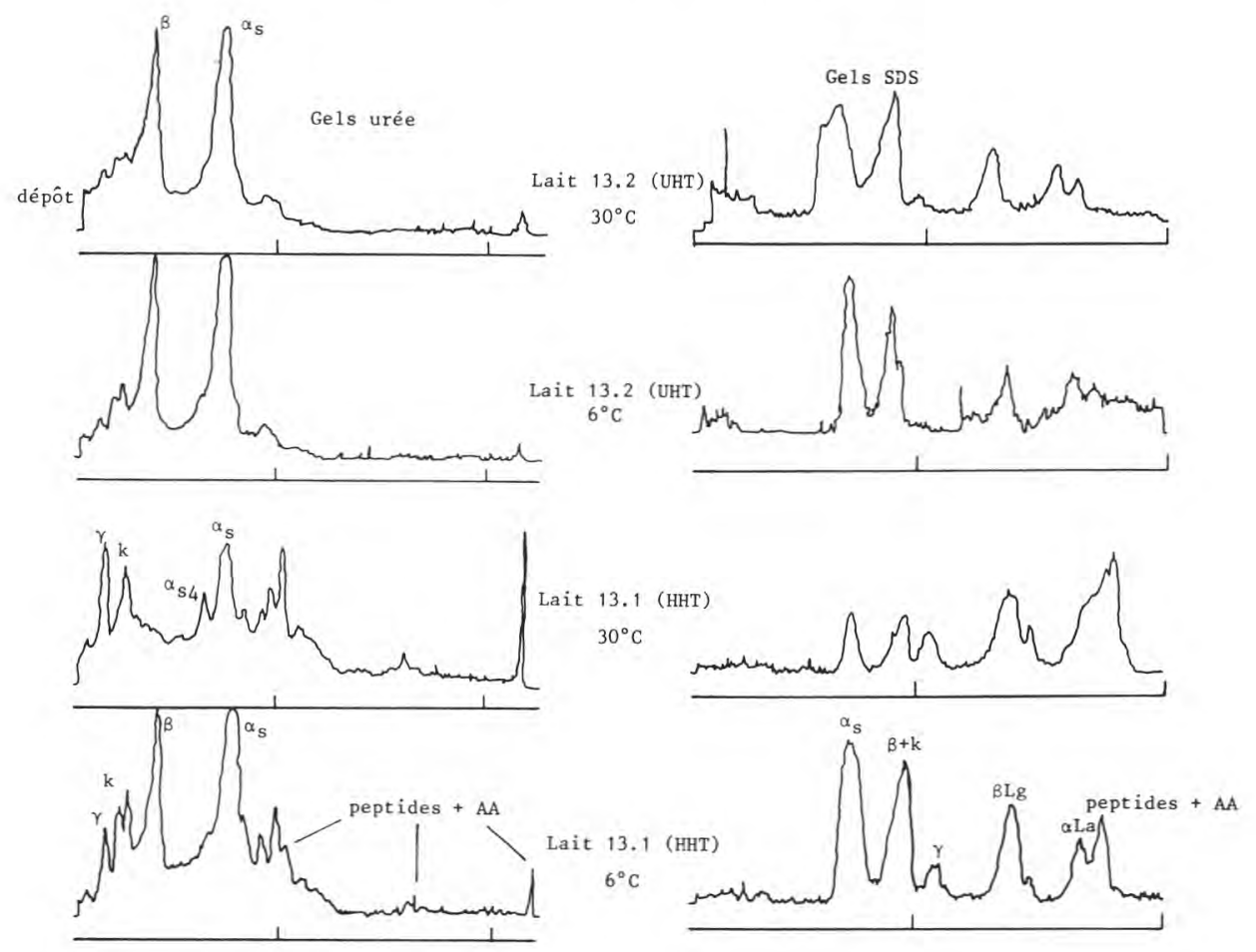

Fig 12. Composition du lait de 13 à 20 jours : influence de la température et du type de traitement. Milk composition at 13 and 20 days: influence of temperature and type of treatment.

dans une moindre mesure. Le lait 13 est cependant un lait peu chargé en germes psychrotrophes; il est possible que pour un lait plus contaminé l'action des enzymes correspondantes soit plus importante. Des expérimentations complémentaires devraient être menées dans ce sens. On peut cependant conclure que les 2 types d'enzymes sont responsables de la protéolyse.

La figure 14 détaille l'évolution du lait à $6{ }^{\circ} \mathrm{C}$. Elle confirme que la protéolyse est moindre, mais qu'elle n'est vraiment négligeable que jusqu'à 20 jours de conservation.

Afin de déterminer exactement les potentialités du traitement HHT pour la pro- duction du lait de consommation, il conviendrait de répéter des essais sur un lait non trié suivi d'un stockage réfrigéré, afin de fixer la durée de conservation conduisant à une bonne qualité organoleptique. De plus, un traitement d'inactivation postérieur à la stérilisation tel que proposé par Hill (1988) devrait être testé, afin de déterminer s'il pourrait permettre une conservation à $30^{\circ} \mathrm{C}$, sans dénaturation excessive des protéines solubles.

\section{CONCLUSION}

Un nouveau dispositif d'injection de vapeur a été testé; il permet de réchauffer un liquide de $80^{\circ} \mathrm{C}$ à $160^{\circ} \mathrm{C}$ en $0,15 \mathrm{~s}$. 

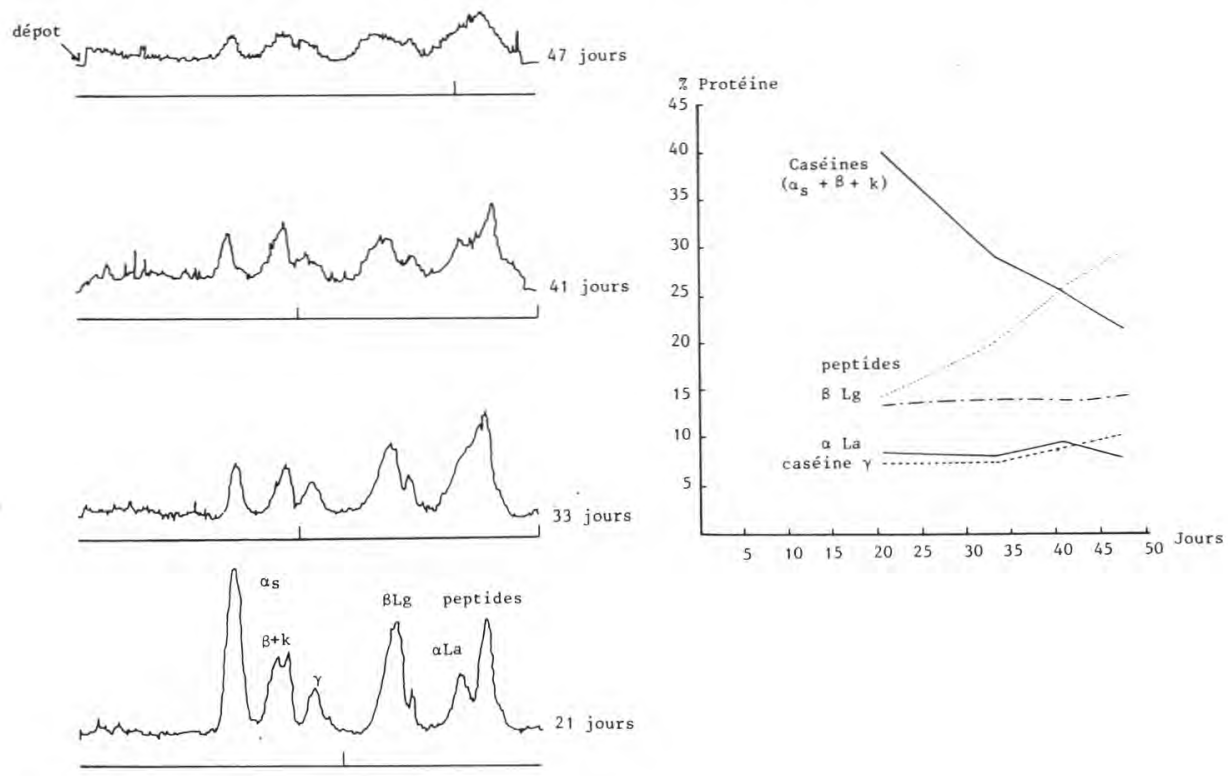

Fig 13. Évolution du lait HHT 13.1 stocké à $30^{\circ} \mathrm{C}$. HHT No 13-1, evolution for storage at $30^{\circ} \mathrm{C}$.

Ce dispositif a été appliqué au lait, avec des temps de chambrage à $160^{\circ} \mathrm{C}$ de 0,2 $s$ à $0,4 \mathrm{~s}$, avec l'objectif de mettre au point un nouveau procédé de stérilisation, dite stérilisation Hyper-Haute-Température, préservant mieux la qualité du lait que le traitement UHT.

II a été constaté que le traitement permettait bien d'obtenir la stérilité. Les protéines solubles sont moins dégradées que dans le cas d'un traitement UHT indirect. La dénaturation de ces protéines est cependant bien supérieure à ce que laissait supposer le modèle utilisé. On a fait des hypothèses concernant cet écart, que des expérimentations complémentaires devraient vérifier.

Cependant la teneur en protéines solubles n'est pas significative de la qualité organoleptique des laits testés, puisque des dégustations ont montré que le lait traité par HHT était significativement différencié du lait UHT et n'était pas différencié du lait pasteurisé. L'effet semble cependant masqué par la teneur en matière grasse du lait, ce qui resterait à confirmer.

Pour ce qui concerne la conservation du lait HHT, le problème majeur rencontré a été le développement d'une protéolyse très importante sur les laits conservés à $30^{\circ} \mathrm{C}$, aboutissant à terme à une transparisation du lait. Cette protéolyse est attribuée à la fois à la plasmine et aux protéases des bactéries psychrotrophes.

L'utilisation d'un lait de bonne qualité bactérienne n'est pas suffisante pour inhiber cette protéolyse. Par contre un stockage réfrigéré permet de la limiter, ce qui laisse penser qu'un lait de qualité proche du lait pasteurisé et se conservant 15 à 30 jours au froid pourrait être produit par le traitement HHT. 


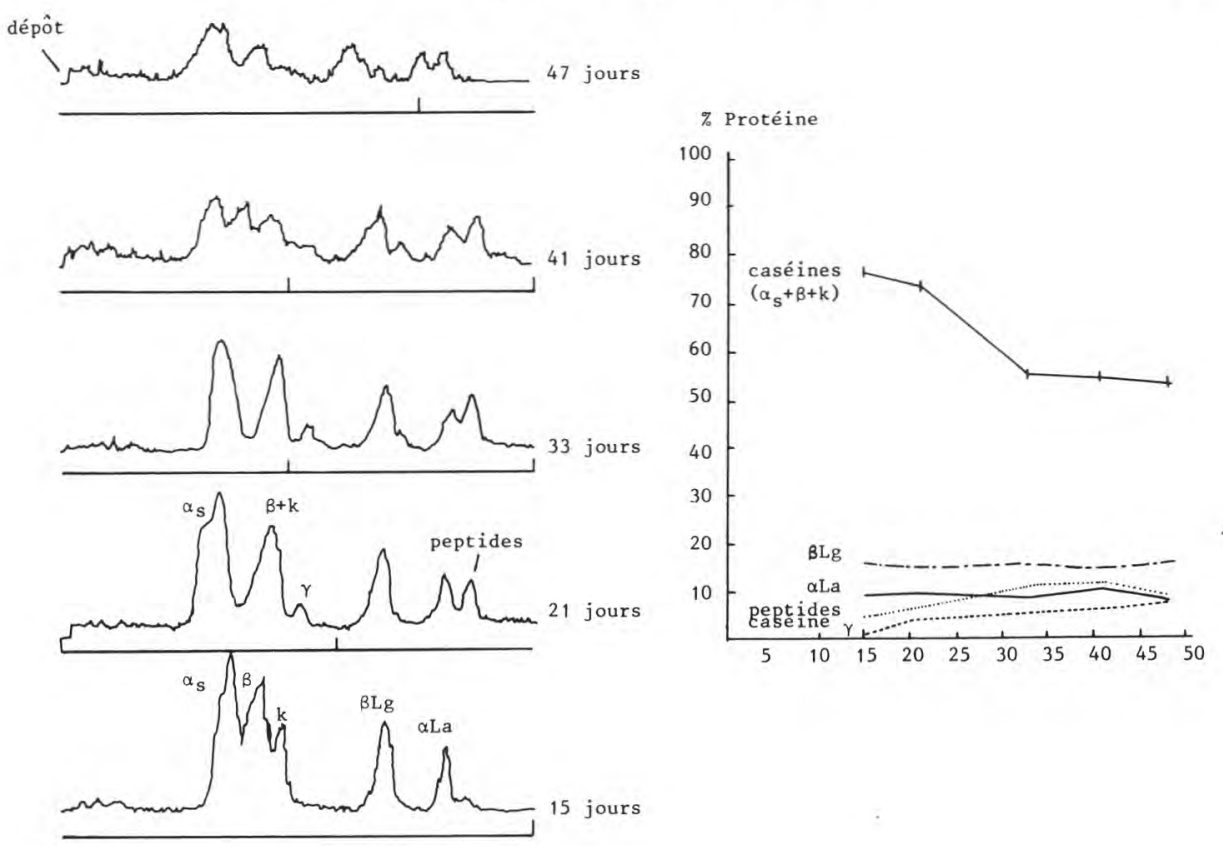

Fig 14. Évolution du lait HHT 13.1 stocké à $6^{\circ} \mathrm{C}$.

HHT No 13.1, evolution for storage at $6^{\circ} \mathrm{C}$.

Pour ce qui concerne la possibilité d'une conservation à $30^{\circ} \mathrm{C}$, un traitement d'inactivation des protéases à basse température postérieur au traitement HHT devrait être testé, à la condition que la température soit suffisamment basse pour ne pas dénaturer les protéines solubles.

\section{REMERCIEMENTS}

Nous remercions vivement le Centre de recherche de la SODIMA, en particulier M Loones et M Selaries et I'ULN, en particulier Mme Quilichini et $M$ Le Treut, pour leur aide sans laquelle les essais sur le lait n'auraient pu être réalisés. Nous remercions également M Cerf, directeur du laboratoire de génie de l'hygiène et des procédés alimentaires de l'INRA pour les précieux conseils qu'il nous a prodigués tout au long de cette étude.

\section{RÉFÉRENCES}

Burton H (1977) The direct heating process for the UHT sterilization of milk. Chem Eng Nov, 792-795

Cerf O (1982) Influence du recyclage du lait sur les caractéristiques du lait UHT. Rev Lait Fr 405, 15-17

Chawla JM (1977) Offenlegungsschitt 2627880 (Brevet d'invention) DT 2627880 A 1 (29-121987)

Clift R, Grace JR, Weber ME (1978) Bubble, Drops and Particles, Academic Press, New York 
Dannenberg F (1986) Zur Reaktionskinetik der Molkenprotein denaturierung und deren technologischer Bedentung. Thèse de DocteurIngénieur, Université technologique de Münich

Dannenberg F, Kessler HG (1988) Reaction kinetics of the denaturation of whey proteins in milk, J Food Sci 53, 1, 258-263

Daudin JD, Cerf O (1977) Influence des chocs thermiques sur la destruction des spores bactériennes par la chaleur Lebensm Wiss Technol 10, 203-207

Fabre C (1982) Étude d'un système de condensation rapide de vapeur dans un liquide en écoulement pour la stérilisation du lait. Thèse Docteur-Ingénieur, INPL, Nancy

FIL (1962) Determination of the total nitrogen content of milk by the kjeldahl method. FILIDF 20

Helaine E (1981) Le lait UHT : un produit de qualité longue conservation. Rev Lait Fr 393, 13-22

Hetsroni G (1982) Handbook of Multiphase Systems. Hemisphere Publishing Corporation, Washington
Hill AR (1988) Quality of Ultra-High-Temperature processed milk. Food Technol 42, 9, 92-97

Ng-Kwai-Hang KF, Kroeker EM (1984) Rapid separation and quantification of major caseins and whey proteins of bovine milk by polycrylamide gel electrophoresis. J Dairy Sci $67,3052-3056$

Laemmli VK (1970) Cleavage of structural proteins during assembly of head of bacteriophage T4. Nature 227, 680

Lyster RLJ, Hillier PM (1979) Whey protein denaturation in heated milk and cheese whey. $J$ Dairy Res 46, 219-220

Perkin AC (1985) Review of UHT processing methods. J Soc Dairy Technol 38, 69-73

Ray (1951) Technologie laitière, Dunod, Paris

Trossat D (1980) Réalisation et étude des caractéristiques d'un appareil de stérilisation du lait de type UHT à injection directe de vapeur. Thèse de Docteur-Ingénieur, ENSAM, Paris

Walstra P, Jenness R (1984) Dairy Chemistry and Physics. John Wiley \& Sons, New York 